\title{
Challenges of Cervical Cancer Screening Among Women of Reproductive Age in Kisii Town, Kisii County, Kenya
}

\author{
Robert M. Kei ${ }^{1}$, Julius K. M’Ndegwa ${ }^{2}$, Taratisio Ndwiga ${ }^{1}$, Faith Masika ${ }^{1}$ \\ ${ }^{1}$ Department of Environmental Health, Moi University, Eldoret, Kenya \\ ${ }^{2}$ Department of Civil Engineering, Moi University, Eldoret, Kenya
}

Email address:

mburugukei@gmail.com (R. M. Kei),jkmndwgwa@yahoo.co.uk (J. K M’Ndegwa), taratisiondwiga@yahoo.com (T. Ndwiga), fmasika@gmail.com (F. Masika)

\section{To cite this article:}

Robert M. Kei, Julius K. M'Ndegwa, Taratisio Ndwiga, Faith Masika. Challenges of Cervical Cancer Screening Among Women of Reproductive Age in Kisii Town, Kisii County, Kenya. Science Journal of Public Health. Vol. 4, No. 4, 2016, pp. $289-296$. doi: $10.11648 /$ j.sjph.20160404.14

Received: May 17, 2016; Accepted: June 3, 2016; Published: June 17, 2016

\begin{abstract}
Introduction: Cervical cancer is a preventable non communicable disease of public health importance. It is the most common genital cancer and one of the leading causes of death among female population. Cervical cancer is a prevalent yet preventable cause of death among Kenyan women. The main objective of this study was to assess the knowledge, practice and barriers towards screening for premalignant cervical lesions among women aged 15 years and above years in Kisii Town, Kisii County. Methods: A descriptive cross-sectional study design was used and the sample population was selected among women in Kisii town. Data was collected using a structured interviewer-administered questionnaire. This instrument was pretested in a neighboring town to ascertain validity. Random sampling was used, in order to give every woman in Kisii Town opportunity to be included in the study. The sample size of 151 respondents was realized using Fisher et al, (1998). formula. Data Analysis: The SPSS version 20.0 was used to analyze the data which was presented in prose, figures and tables. Chisquare test was used to measure the strength of associations between the various variables where a $p$-value of $=$ or $<0.05$ was considered to be statistically significant. Results: Knowledge on cervical cancer and screening was very low among women in Kisii Town, because $20.5 \%$ knew about vaginal bleeding and $15 \%$ knew about having multiple sexual partners as a risk factor. There is relationship between the level of education and belief on the cure for cancer $(\mathrm{P}=0.000)$, those who are more educated believed that cervical cancer can be cured at early stages while those with less education believed that cancer cannot be cured. Conclusion and Recommendation: Knowledge on cervical cancer and screening was low, hence poor practice on screening among women. Main barriers for not screening were ignorance and fear of positive results. It may be recommended that cancer screening machines should be purchased by County government and distributed to town health facilities at subsidized charge for screening. Also, awareness creation including counseling should be intensified in order to promote screening compliance.
\end{abstract}

Keywords: Cervical Cancer, Cancer Screening, Human Papilloma Virus, Cancer Lesions

\section{Introduction}

Cervical cancer is the most common genital cancer and one of the leading causes of death among female population. Cervical cancer is a prevalent yet preventable cause of death among Kenyan women. Lack of feasible vaccination, screening and treatment options combined with a high incidence of risk are the main causal factors [1].

According to the National Cervical Cancer Prevention Program strategic plan of 2012-2015, cervical cancer is a major public health concern in Kenya. It is the second most common cancer in women but the most common cause of cancer deaths. Although it is easily prevented through behavior change, vaccination, screening, early detection and treatment of precancerous lesions, most eligible women of reproductive health in Kenya have never been screened. Consequently, many cases present late when treatment is more difficult and more expensive to obtain [2]. It is a major global health problem, with nearly 500,000 new cases occurring each year worldwide. Each year an estimated 270,000 women die from the disease with nearly $80 \%$ of the 
deaths occurring in developing countries. It is the leading killer disease of women in the developing world and is the second most common malignancy in women worldwide after breast cancer. Invasive cervical cancer is the most common cancer in women worldwide, but fortunately this cancer is preventable by screening for premalignant lesions but this is rarely provided and hardly utilized [3]. Furthermore, infection with Human Immunodeficiency virus (HIV) increases women's risk for Human Papilloma virus (HPV) infection, cervical cancer precursor lesions and invasive cancer. Two thirds (2/3) of the world's cases of HIV infection are found in sub-Saharan Africa, where a shortage of resources and biological factors work synergistically to increase women's lifetime risk of developing cervical cancer. As a result, cervical cancer prevention is a public health priority in sub-Saharan Africa, especially among HIVinfected women [4].

In Kenya cervical cancer is the second most prevalent cancer among women after breast cancer and its incidence is increasing, this is according to [5]. Factors contributing to high risks of cervical cancer in Kenyan women include; multiple pregnancies, early age at first intercourse, hormonal contraceptives, smoking and HIV infection [1].

Kenya has a population of 10.32 million women ages 15 years and older who are at risk of developing cervical cancer. Current estimates indicate that every year 2454 women are diagnosed with cervical cancer and 1676 die from the disease. Cervical cancer ranks as the $2^{\text {nd }}$ most frequent cancer among women in Kenya and the $2^{\text {nd }}$ most frequent cancer among women between 15 and 44 years of age. About $38.8 \%$ of women in the general population are estimated to harbor cervical cancer HPV infection at a given time and $60.9 \%$ of invasive cervical cancers are attributed to HPVs 16 or 18 [6].

Cervical cancer remains the leading malignancy among women in Kenya. The World Health Organization (WHO) documented Kenya as among countries that have the highest incidence of cancer of the cervix in sub-Saharan Africa [7]. It is therefore one of the most important public health problem yet to be recognized as such in sub-Saharan Africa [8].

Studies in Africa and especially Sub-Saharan Africa indicate poor knowledge of cervical cancer as on screening for premalignant cervical lesions [9]. Screening has shown to effectively reduce the incidence of this malignancy in developed countries but in developing countries screening coverage is still low ranging from $2.0 \%$ to $20.2 \%$ in urban areas and $0.4 \%$ to $14.0 \%$ in rural areas [7].

Cervical cancer causes pain, suffering, economic loses and death among many women in the world. Grim statistics show that over 60 Kenyans die of cancer and its related complications every day. It has been shown that cancer in Kenya is the third leading cause of death, killing more people than HIV and Malaria combined [10].

Knowledge of the disease is important, so that people are aware and through motivation they can have positive attitude towards screening for premalignant cervical lesions.

Information obtained from this study may alert Ministry of Health that appropriate measures could be taken to save the lives of Kenyan women by educating them and provide screening services at subsidized cost.

In addition the study will help the Ministry of Health in policy making and planning, for instance towards regular screening of women and availing vaccinations on time. Moreover, several studies indicate that many African nations have not recognized cervical cancer as a disease of public health importance, this study therefore aims at alerting the Ministry of health at the County and National level on the severity of cervical cancer and that prevention should be given priority just like communicable diseases such as T. B and Malaria.

The research project was done in Kisii Town which is the Headquarters of Kisii County and is one of the 47 counties of Kenya. It shares borders with Nyamira County to the North East, Narok County to the south and Homabay and Migori counties to the west. It covers an area of 1032 sq. km with a population of about 1,152,282 people as per the 2009 census. The high and reliable rainfall coupled with moderate temperatures is suitable for growing crops like tea, coffee, pyrethrum, maize, beans, bananas as well as dairy farming [11]. The Abagusii are the main inhabitants of Kisii although other tribes like the Luo and Luhyia have also migrated into the region. Kisii'County is served with various institutions of higher learning, like Kisii University, Gusii Institute of Technology and other satellite campuses of various universities. The county has many health care facilities, both private and public. Kisii Level 5 hospital is the main Referral hospital in the region with a catchment of about 3 million people and staff establishment of about 500 workers and 13 specialists. Oresi health center is a government health facility located within town. The main private hospitals include RAM and Oasis hospitals [12]. There was inadequate cancer screening machines for town health facilities notwithstanding the high cost of screening. The method of screening used in the health facilities was the VIA and VILI and the women should go foe screening within 1-3years,

The main objective of this study was to assess the knowledge, practice and barriers towards screening for premalignant cervical lesions among women aged 15 years and above in Kisii Town, Kisii County.

\section{Literature Review}

\subsection{Knowledge of Women on Cervical Cancer}

In many developing countries, women's knowledge of cervical cancer and Pap smears is very limited. In a survey performed in Nigeria, 254 women were randomly assessed and asked about knowledge of cervical cancer, only $15 \%$ had ever heard of cervical cancer and even less knew about cervical screening [13].

Moreover, a study done in Zimbabwe by [33] shows that of the 514 participants, $91 \%$ had never heard about cervical screening, and $81 \%$ had no previous knowledge of the cervical screening tests. Despite never having had cervical screening and lacking prior knowledge of its purpose, $80 \%$ of 
the females expressed positive beliefs about cervical screening tests after an educational intervention.

According to a study done in Kisumu Kenya only $6 \%$ of the women interviewed had knowledge about cervical cancer. These were women who had at least secondary education and earned a monthly income of more than Ksh 3,000 [14].

Another study conducted in Tanzania (2011) revealed that $78.6 \%$ of women in developing countries had poor knowledge on cervical cancer while $46.3 \%$ had no idea on the symptoms of the disease. This is contrary to the developing countries where most women are aware of cervical cancer and screening services are widely available and utilized [15].

Ever since the concept of cervical smears was first described by Papanicolaou and Traut in 1941, both the incidence and mortality of cervical cancer have markedly decreased in most developed countries, mainly due to effective screening programs [16]. Although there is overwhelming evidence that cervical cancer today is preventable through screening and treatment of premalignant lesions, the service is unfortunately not readily available to the general population in most developing countries including Kenya [17].

From the foregoing, it can be noted that very few women in sub-Saharan Africa are ever screened for cervical cancer. Low levels of awareness and poor knowledge of cervical cancer coupled with unavailability and inaccessibility of cervical cancer screening services are responsible for the very small number of women being screened in sub-Saharan Africa and in other developing countries [18].

In developed countries people seem to be aware of cancer and screening services are widely available and utilized. In a cross-sectional survey of 650 women $15-78$ years of age randomly recruited at 2 hospitals in London, England, 76.2\% perceived cervical cancer to be a common disease and there was good awareness of the association between this cancer with smoking and the number of sexual partners. Furthermore, $91.7 \%$ believed cervical cancer could be treated if detected early enough [18].

A study done in Malaysia on women aged 21-56 years and who had never had a Pap smear test, with the aim to explore their knowledge and awareness of cervical cancer and its screening, showed that there is a lack of knowledge on cervical cancer as well as lack of knowledge on the Pap smear test. Many women did not have a clear understanding of the meaning of an abnormal cervical smear and the need for the early detection of cervical cancer. Many believed that the purpose of the Pap smear test was to detect existing cervical cancer, leading to the belief that Pap smear screening is not required because the respondents had no symptoms. Despite considerable awareness of a link between cervical cancer and sexual activity, as well as the role of a sexuallytransmitted infection, none of the respondents had heard of the human papillomavirus [19].

In Kuwait in one study done regarding cervical cancer screening among Kuwaitian women found that the knowledge about the test was adequate in 147 (52.3\%) women only [20].

In Africa A study done in Cameroon to assess the knowledge of cervical cancer by women living in Maroua, the capital of the Far North Province of Cameroon showed that, of 171 women studied, only 48 (28\%) had prior knowledge of cervical cancer (Tebeu et al, 2008) In a study done in Lagos, $81.7 \%$ of 139 patients with advanced cervical cancer had never heard of cervical cancer before, and $20 \%$, $30 \%$ and $10 \%$ respectively thought the symptoms they had were due to resumption of menses, lower genital infection and irregular menses [21].

In Nigeria, a cross sectional study done in the General outpatient, department of a tertiary hospital in Ibadan, Nigeria, women aged 20 to 65 years attending or visiting the GOP department in a University Teaching Hospital were studied. Of the respondents, only $15 \%$ had heard of cervical cancer [8].

A cross-sectional survey among college women in a university in Ghana showed that only $7.9 \%$ were aware of the link between human papilloma virus and cervical cancer [22]. Knowledge is also poor among health professionals where in Niger a survey of 144 female health professionals at two referral hospitals with facilities for Pap smear showed that twenty two percent could not list any risk factor for cervical carcinoma [23].

\subsection{Practice of Women on Cervical Cancer Screening}

Practice towards screening for cervical carcinoma is poor to even those with knowledge of the disease and knowledge on the importance of screening. The incidence of cervical cancer has declined in the developed nations due to routine use of cervical screening services. In the developing nations opportunistic screening is the practice and many women present with late stage disease [24]. In developed countries majority went for screening as compared to developing countries. In a cross-sectional survey of 650 women 15-78 years of age randomly recruited at 2 hospitals in London, England. 80.5\% of these women had at least one Pap smear and $71.5 \%$ reported regular smears (every 3-5 years) [19].

Practice on screening among Africans has been poor as shown in a study done in South Africa, in spite of knowledge of cervical screening and the availability of such services, majority of women $(87 \%)$ from higher social and educational backgrounds did not undergo cervical screening. Most patients resided within a 12 kilometer radius of a facility that either provided or could potentially provide screening [25].

In one study in Nigeria where 144 questionnaires were filled only $5.7 \%$ had ever undergone a pap smear [26]. This is also supported by Nwankwo K. C et. al in their study conducted in Nigeria in 2011 which also revealed poor practice on cervical screening and knowledge whereby only $15.5 \%$ were aware of the availability of cervical screening services, and this awareness significantly varied with the level of education attained $(\mathrm{p}<0.0001)$. Moreover only $4.2 \%$ of the respondents in the study had ever done a Pap smear test [24]. 


\subsection{Barriers to Cervical Cancer Screening}

Practice towards cervical screening has been shown to be poor especially in sub-Saharan Africa.

In sub-Saharan Africa knowledge and awareness of cervical cancer and screening is poor and mortality from the disease still very high. Much of the recent research into women's knowledge of cervical cancer and screening has been conducted either in developing countries or among ethnic minorities in developed countries. Such studies usually identify low levels of knowledge, which, it is believed contribute to low rates of screening uptake in these populations [9].

Inadequate knowledge and lack of awareness are barriers to cervical cancer prevention. Many participants in previous screening studies revealed that they had little knowledge of cervical cancer screening [20].

A study done in Malawi found that the primary cue to action for cervical cancer screening was symptoms of cervical cancer. Major barriers to seeking preventative screening included knowledge levels, low perceived susceptibility and low benefits from the service Study participants did not view cervical cancer screening as critical health care. Interviews suggested that use of the service could increase if women are recruited while visiting the hospital for a different service [27].

Cultural beliefs and attitudes towards cancer have also been cited as barriers to cervical cancer screening by [28]. According to their study the Black Minority Ethnic groups (BME) associated cancer with certain death and felt it was not talked about within their communities. There was a superstition that talking about it would enhance its malevolent power.

Participants in a study done in Asia admitted that they did not go for cervical screening because of fear of pain and discomfort during screening process. Respondents assume that this physical test will cause pain as the test kit has to be inserted into the vagina in order take samples Other researchers reported that rumors spread by their close family members or friends that the screening process was painful [29], [20].

Researchers have reported previously that embarrassment influences cervical cancer screening uptake using Pap smear method. Most women feel uncomfortable with the idea of vaginal examination for 'private parts'. Previous studies in the US reported that $31 \%$ of Hispanic women undertook the Pap smear test admitted that the process was embarrassing, while $60 \%$ who did not present for screening also stated the same. Screening by male doctors affected women's decision in presenting for examination [20]. This is supported by another study which revealed that embarrassment and pain are barriers to cervical cancer screening [30].

Again, a study conducted in 2011 by Nkwakwo revealed that the most important factors hindering the use of cancer screening services were lack of knowledge (49.8\%) and the feeling that the women had no medical problems (32\%) [24].

\subsection{Problem Statement}

Despite the existence of a previous National Cervical
Cancer Prevention Strategic Plan -NCCPSP, 2002-2006, implementation of the national screening program is still low and haphazard. Cervical cancer screening occurs in only a few selected sites and in disjointed projects rather than a fully-fledged national level program. This explains why screening coverage is still low at 3.2\% [31].

Not much is known about Kenyan women knowledge, practice and barriers towards cervical cancer and screening for cervical lesion. This study aims at investigating how knowledgeable these women are, what is therefore their practice and barriers on screening for cervical premalignant lesions which is the key to prevention of overt carcinoma of the cervix.

\section{Methodology}

Data was collected using a structured intervieweradministered questionnaire. This instrument was pretested in a neighboring town to exclude ambiguities. The questionnaire had items on socio-demographic details, knowledge practice and barriers to cervical cancer screening on women attending $\mathrm{MCH}$ services. The questionnaire was administered to all volunteer women who fulfilled the inclusion criteria.

A descriptive cross-sectional study design was used as this provided a snap shot of knowledge, practice and barriers to cervical cancer screening for all the women of reproductive age in Kisii Township. Those women who were too sick, to understand what the study was all about and women with the history of the disease (carcinoma of the cervix) were excluded from the study.

Sample size was calculated using [32] formula where a sample of 151 respondents was obtained.

Systematic random sampling technique was employed in the study. This gave an equal chance for all women of reproductive age that was included in the study. The doctor and nurse in-charge of the cervical cancer clinic were purposively chosen as key informants because they were the ones with first-hand information on cervical cancer.

The data was then coded before being entered into the computer for analysis using Statistical Package for the Social Sciences (SPSS) version 20. Chi-square test was used to measure the strength of associations between various variables where a $\mathrm{p}$-value of $=$ or $<0.05$ was considered statistically significant.

\section{Results}

Majority of the respondents (58.9\%) were between 20-30 years old and $(0.7 \%)$ was between $61-70$ years. From the records in Kisii level 5 Hospital, majority of women who were testing positive for cervical cancer were in the age group of 31 years and above. Most, (36.4\%) of the respondents had attained secondary education, (32.5\%) primary education and (29.8\%) had attained university/college level.

Majority, (60.3\%) were in monogamous marriages, and 
$(23 \%)$ were single. The marital status of the respondents is dependent on the level of education $(\mathrm{PV}=0.000)$ those who have attained higher level of education are in monogamous marriages and those with less educational qualifications were in polygamous marriages.

In addition, (37.1\%) of the respondents engaged in business activities to earn their living, $(27.1 \%)$ were farmers and $(19.9 \%)$ did not have a source income as they were still students. Majority $(84.1 \%)$ had heard about cervical cancer and $(15.9 \%)$ had not heard about cervical cancer, $(39.7 \%)$ got the information on news media (radio and television), $(29.2 \%)$ from health workers and (1.3\%) from the internet. From the study, $55 \%$ of the respondents did not know the symptoms of cervical cancer, $(20.5 \%)$ knew about vaginal bleeding and (15\%) knew about vaginal foul discharges. Other types of symptoms mentioned by the respondents included wounds in the vagina and itching.

\subsection{Knowledge on Risks to Cancer}

Most $(63 \%)$ of the respondents did not know the risk factors for cervical cancer, (15\%) knew about risk of having multiple sexual partners and (11.3\%) knew about acquiring HPV virus. The main risk factors for cervical cancer included early sexual intercourse and having multiple sexual partners. Again, (68.2\%) of the respondents believed that cancer can be cured while $(17.9 \%)$ said it could not be cured and (13.9\%) did not know. However, (21.2\%) said that it could be cured by specific drugs given in hospital, by radiotherapy $(19.9 \%)$, through surgery $(9.3 \%)$ and $(4.6 \%)$ believed that cervical cancer could be cured through herbal medicine. There is a relationship between the level of education and cancers being cured $(\mathrm{PV}=0.000)$, those who are more educated believe that cervical cancer can be cured at early stages while those with less education believed that cervical cancer cannot be cured.

Forty eight percent $(48.5 \%)$ of the respondents believed that cervical cancer treatment in Kenya is very expensive and beyond the reach of an average Kenyan while (30.5\%) did not know about the cost of cervical cancer treatment. Breast cancer was the most known type of cancer (59.1\%), followed by prostate and throat cancer at $(8.7 \%)$. The respondents who had heard about cervical cancer screening were $(64.2 \%)$ and (35.8\%) had not heard about it. However, (39.1\%) did not know who should be screened, (30.5\%) believed that all sexually active women should be screened, (39.7\%) of the respondents did not know how frequent should one go for screening and (26.6\%) thought it could be done yearly while $(17.9 \%)$ said that it should be done monthly. Again, (63.6\%) did not know the screening methods, $(28.5 \%)$ knew about Pap smear.

Of the $(16.6 \%)$ who had been screened, $(6.7 \%)$ had been screened within the last two weeks, $(5.3 \%)$ one year ago, and $(3.3 \%)$ more than 3 years ago and (1.3\%) in the last 3 months.

\subsection{Reasons for not Screening}

The respondents felt that they are healthy and they did not need to go for screening were $(18.1 \%)$ that $(16.5 \%)$ were not aware of screening, (15\%) were not decided to go for screening and (13.4\%) were afraid of the screening results.

\subsection{Barriers to Screening}

Majority were not aware of the importance of screening due to ignorance (30.5\%)-others were affected by fear of screening outcome (28.5\%), Shyness (9.9\%), Expensive (6.6\%) and Painful (7.5\%).

\section{Discussion}

In this study $84.1 \%$ of the respondents had heard about cervical cancer and their main source of information was the news media (39.7\%) and health workers (29.4\%). Similarly in a study done in Nigeria by Anorlu among women aged 2065 in the General Outpatient department in a university teaching hospital only $15 \%$ of the respondents had heard about cervical cancer [8].

When asked about the symptoms of the disease, majority (55\%) of the respondents don't know any symptoms cervical cancer but at least $20.5 \%$ could identify vaginal bleeding and $15 \%$ foul vaginal discharges which are the main symptoms of cervical cancer. Other symptoms that were mentioned included wounds and itching of the vagina which are not symptoms of cervical cancer but symptoms of infections like urinary tract infections. More over most (63\%) did not know any risk factors of cervical cancer, only $15 \%$ knew about having multiple sexual partners, $5.3 \%$ knew about early sexual intercourse and $4 \%$ of the respondents associated cervical cancer to smoking of cigarettes. Likewise a study by John James in Tanzania revealed that $78.6 \%$ of women in developing countries had poor knowledge on cervical cancer while $46.3 \%$ had no idea on the symptoms of the disease. This is contrary with the developing countries where most women are aware of cervical cancer and screening services are widely available and utilized [15].

In this study $68.2 \%$ of the women interviewed believed that cervical cancer can be cured and only 17.9 did not believe that cancer can be cured at early stages. Of those who believed that cervical cancer can be cured at early stages, $21.2 \%$ said that it can be treated by specific drugs given in hospitals, $19.9 \%$ said that cancer can be cured through radiotherapy and $4.6 \%$ through herbal medicines. The findings of the study done by [19] also revealed the same whereby $91.7 \%$ of the respondents believed that cancer can be treated if detected early [19].

As much as $84 \%$ have heard about cervical cancer only $64 \%$ have heard about cervical cancer screening. Out of the $64 \%$ who have heard about cervical cancer screening, $39.1 \%$ don't know who should be screened, but at least $30.5 \%$ believe that it's the sexually active women who should be screened.. All sexually active women should go for cervical cancer screening but at Kisii level 5 hospital women above 20 years were the ones going for screening. When asked about how frequent the screening should be done $39.5 \%$ didn't know while $26.6 \%$ said that screening should be done 
after one year.. Very few women know about the screening methods used, only $28.5 \%$ knew about pap smear while the majority $63.6 \%$ don't know any screening method used. The method of screening used in Kisii health facilities was the VIA and VILI. In another study done in Kuwait, by [21] in 2009 only $52.3 \%$ of the women there had knowledge on cervical cancer. Moreover a study done in Zimbabwe by [33] showed that of the 514 participants, $91 \%$ had never had cervical screening, and $81 \%$ had no previous knowledge of the cervical screening tests. Despite never having heard of cervical screening and lacking prior knowledge of its purpose, $80 \%$ of the females expressed positive beliefs about cervical screening tests after an educational intervention. In Cameroon a study done among women in Maroua, the capital in the far north by [34] to assess knowledge of cervical cancer showed that out of 171 women studied only 48 (28\%) had prior knowledge on cervical cancer. In Kisii county many women have heard about cervical cancer and screening but very few seem to be aware of the symptoms (20.5\%) knew about vaginal bleeding and $15 \%$ knew about vaginal foul discharges), risk factors ( $4 \%$ associated cervical cancer to smoking, 5.3\% early sexual intercourse and 15\% multiple sexual partners) and even treatment of the disease. This is valiant with the developed countries, where people seem to be aware of cancer and screening services are widely available and utilized [19] did a cross-sectional survey of 650 women $15-78$ years of age randomly recruited at 2 hospitals in London, England, $76.2 \%$ perceived cervical cancer to be a common disease and there was good awareness of the association between this cancer with smoking and the number of sexual partners.

In this study $16.6 \%$ of the respondents have been screened for cervical cancer, of those that have been screened, only $4 \%$ have done it more than once and $12 \%$ have been screened once. Moreover $6.7 \%$ were screened within the last two weeks, $5.3 \%$ in the last one year and $3.3 \%$ more than 3 years ago. From this study, we find that despite many being aware of cervical cancer and screening very few have put what they have heard into action. Similarly Wellensiek in his study in south Africa found out that practice on screening among Africans was poor in spite of knowledge of cervical cancer screening and the availability of such services, majority of women $(87 \%)$ from higher social and educational backgrounds did not undergo cervical screening and Most patients resided within a 12-kilometer radius of a facility that either provided or could potentially provide screening [24]. Moreover a study by Udigwe in Nigeria had similar results with the findings of this study whereby only $5.7 \%$ of the 144 study participants had undergone cervical cancer screening [26].

The findings of this study are at valiant with the findings of [19] who found out that, in developed countries majority went for screening as compared to developing countries. In a cross-sectional survey of 650 women 15-78 years of age randomly recruited at 2 hospitals in London, England. 80.5\% of these women had had at least 1 Pap smear and $71.5 \%$ reported regular smears (every 3-5 years) [19] and they concluded that practice towards screening for cervical carcinoma is poor to even those with knowledge of the disease and knowledge on the importance of screening.

When the respondents who have not gone for screening were asked why they haven't gone, the majority $18.1 \%$ said that they were healthy and they did not have a reason to go for screening, $16.5 \%$ stated that they were not aware of cervical cancer screening, $15 \%$ had not decided when to go, while another $15 \%$ said that the screening procedure was painful and $13.4 \%$ were afraid of screening results. The results of this study are supported by a similar study conducted in Nigeria in 2011 by [24], which showed that the most important factors hindering the use of cervical cancer screening services were lack of knowledge (49.8\%) and the feeling by women that they have no medical problems (32\%).

Majority $(30.5 \%)$ of them said that most women did not know about cervical cancer screening [21] also found out that lack of adequate knowledge and lack of awareness can become barriers to cervical cancer prevention [28] in his study conducted in Malawi also revealed that the major barriers to preventative screening included low levels of knowledge, low perceived susceptibility and low benefits from the service. $28.5 \%$ of the respondents stated that most women did not go for screening because they are afraid of being found with the disease. From the key informant interview, it was revealed that most women did go for screening because they are afraid of the screening results.

Almost ten percent $(9.9 \%)$ of the respondents said that most women felt shy exposing themselves for screening. Austin in his study in 2002 discovered that most women feel uncomfortable with the idea of vaginal examination of 'private parts' [35]. Likewise Byrd in 2004 in Asia concealed that $31 \%$ of Hispanic women who undertook a pap-smear test admitted that the process was embarrassing, while $60 \%$ who did not present for screening also stated the same and added that screening by male doctors affected women's decision in presenting for examination [30] also established that barriers to screening were embarrassment and pain.

Sixty six $(6.6 \%)$ said that some did not go for screening because it was expensive. In Kisii level 5 Hospital, as cervical screening was done at a cost of Ksh. 300 which was beyond several people's daily income in this county.

Lastly $7.5 \%$ did not go for screening because they believed that the procedure was painful. Similarly a study conducted in Asia in 2004 by Byrd established that women don't go for screening because of fear of pain and discomfort during the screening process [20] also in their study discovered that respondents assume that the physical test will cause pain as the test kit has to be inserted in the woman's vagina in order to take sample.

\section{Conclusion}

Cervical cancer continues to be a major public health problem in Kenya. This study revealed the limited knowledge about cervical cancer and low rate of screening for premalignant cervical lesions. It was established that 
many women have heard about cervical cancer and screening but very few have gone for screening. This shows that their knowledge did not translate to practice.

The findings highlighted lack of knowledge and information on factors that may have contributed to women's non-attendance at screening. It is not surprising that the women's reluctance to undergo cervical cancer screening appears to be based on lack of knowledge about the cervical cancer, the risk factors of cervical cancer and also lack of knowledge on the eligibility and availability of screening services. Perhaps education, communication and reassurance are required to overcome such resistances.

The major barriers to screening included lack of awareness, women feeling shy of the screening procedure and some feeling that they are healthy.

\section{Recommendations}

- More cancer screening machines should be purchased by County government and distributed to all town health facilities at a subsidized charge for screening

- Women should be counseled before being screening and awareness should be intensified on the importance of screening in order to promote compliance.

\section{References}

[1] Henley, P. (2012). Preventing preventable cervical cancer in Kenya. Africa portal, Vol. 38 Pp 1-10.

[2] Government of Kenya (2012) National cervical cancer prevention program: Strategic Plan 2012 - 2015. Ministry of public health and sanitation and Ministry of Health.

[3] Saad Aliyu Ahmed, Kabiru Sabitu, Suleiman Hadejia Idris, and Rukaiya Ahmed.(2013). Knowledge, attitude and practice of cervical cancer screening among market women in Zaria, Nigeria. Niger Med J. 2013 Sep-Oct; 54 (5): 316-319. doi: $10.4103 / 0300-1652.122337$

[4] Megan J Huchko, Jennifer Sneden, Hannah H Leslie, Naila Abdulrahim, May Maloba, Elizabeth Bukusi \& Craig R Cohen (2013). A comparison of two visual inspection methods for cervical cancer screening among HIV-infected women in Kenya. World Health Organization. 2013. doi: http://dx.doi.org/10.2471/BLT.13.122051

[5] WHO fact sheet No 380 September, 2013

[6] WHO/ICO (2010). Information Centre on HPV and Cervical Cancer (HPV Information Centre). Human Papil-lomavirus and Related Cancers in World. Summary Report 2010.

[7] Karly S, Silvia de sanjose and Philippe M. (2009). Epidemiology and prevention of human papillomavirus and cervical cancer in sub-saharan Africa: Tropical medicine and International Health, 14 (10), 1287-1302.

[8] Anorlu, R. (2008). Cervical cancer: the Sub-saharan African Perspective. Reproductive health matters, Vol. 16, Issue 32, Pp 41-49. DOI: http://dx.doi.org/10.1016/S09688080(08)32415-X

[9] Gichangi P, Estamble B and Bwayo J (2003). Knowledge and practice about cervical cancer and pap smear testing among patients at Kenyatta National Hospital. International journal of Gynecological Cancer, 827-833.

[10] Wangui., J. (2011). Cancer in kenya should not be a death sentence. the WIP article. Article accessed from: http://thewip.net/2011/12/20 on $18^{\text {th }}$ january, 2015.

[11] Kisii County government. Investiments in agriculture. www.kisii.go.ke/index.php/county-profile/position-and-size. Accessed 28/6/2015.

[12] Kisii referal Hospital (2014) Kisii teaching and referral hospital. Retrieved $10^{\text {th }}$ March, 2015, from kisii hospital.org/index.php/2014-03-08-08-28/brief-profile

[13] Ayaji I. O and Adowole I F (1998). Knowledge and Altitude of general outpatient attendants in Nigeria to cervical cancer. The central African Journal of medicine. 1998 Vol. 44 (2), pp 41-43.

[14] Sudenga L Staci, R. F. (2013). Knowledge, Attitude Practice and perceived risk of cervical cancer among kenyan women. KISUMU. sanitation, M. O. (2012). National cancer prevention program strategic plan 2012-2015.

[15] James John (2011). The knowledge, attitude, practice and perceived barriers towards screening for premalignant cervical lesions among women aged 18years and above, in songea urban, Ruvuma, Tanzania. "A dissertation Submitted in partial Fulfillment of the Requirement for the Degree of Master of Medicine (Obstetrics and Gynecology) of the Muhimbili University of Health and Allied Sciences. Tanzania".

[16] Whitfield (1995) "Premalignant and Malignant diseases of the cervi|" In P. K. R., Dewhurst's Text book of Obstetrics and Gynaecology for Post graduates (Pp. 717-737). Oxford: Blackwell science.

[17] Lazcano-Ponce EC, Moss S, Alonso de Ruiz P, SalmerónCastro J, Hernández-Avila M.(1999) Cervical cancer screening in developing countries: Why is it ineffective? The case of Mexico. Arch Med Res 1999; Vol. 30(3): 240-250.

[18] Yu CK, Rhymer J. (1998). "Womens attitudes to and awareness of smear testing and cervical cancer.". (Y. CK, Ed.) Br J Fam Plann, Vol. 23 (4), Pg 123-33.

[19] Wong LP, Wong YL, Low WY, Khoo EM, Shuib R. (2009). Knowledge and awareness of cervical cancer and screening among Malaysian women who have never had a Pap smear: a qualitative study. Singapore Med. Journal. Vol. 50 (1): 49-53.

[20] Al Sairafi, M. and F. A. Mohamed, (2009). Knowledge, attitudes, and practice related to cervical cancer screening among kuwaiti women. Medical Principles and Practice, Vol. 18 (1): Pg 35-42.

[21] Anorlu R. I, Banjo A. A. F., et. al. (2000). Cervical cancer and cervical cancer screening; Level of awareness in women attending a primary health care facility in Lagos. Nigeria post graduate medical journal, Vol. 70 (16).

[22] Abotchie and Shokar (2009) Cervical cancer screening among college students in Ghana: knowledge and health beliefs. Int J Gynecol Cancer. Vol. 19 (3): 412-6.

[23] Anya SE, Oshi DC, Nwosu SO, et al. (2005). Knowledge, attitude and practice of female health professionals regarding cervical cancer and pap smear. Niger J Med, Vol. 14 (3), 283286. 
[24] Nwankwo K. C., Aniebue U. U., Aguwa E. N., Anarado A. N. and Agunwah E. (2011): Knowledge Attitudes and Practices of Cervical Cancer Screening among Urban and Rural Nigerian Women: a Call for Education and Mass Screening. European Journal of Cancer Care, Vol. 20 (3) Pp. 362-367.

[25] Wellensiek N, M. M. (2002). Knowledge of cervical cancer screening and use of cervical cancer screening facilities among women from various socio-economic backgrounds in Durban, Kwazulu Natal, South Africa. International Journal of Gynecology, Vol. 12 (4), 376-382.

[26] Udigwe. (2006). Knowledge, attitude and practice of cervical cancer screening (pap smear). Niger J Clin Pract, Vol. 9 (1), Pg 40-43.

[27] Victoria K Fort, Mary Sue Makin, Aaron J Siegler, Kevin Ault, and Roger Rochat (2011). Barriers to cervical cancer screening in Mulanje, Malawi. Patient Prefer Adherence. Vol. 5: 125-131.

[28] Thomas Veronica, Tariq Saleem, Rachel Abraham (2011). Barriers to effective uptake of cancer screening among black and minority ethnic groups. Research study. International Journal of Palliative Nursing, 2005, Vol. 11, (11), Pp 562-571.

[29] Byrd, T., Peterson, S., Chavez, R., \& Heckert, A. (2004). Cervical cancer screening beliefs among young hispanic women. Preventive Medicine, Vol. 38, 192-197.

[30] Yifru T, and Asherber. (2008). Knowledge attitute and practice of screening for carcinoma of the cervix among reproductive health clients at three teaching hospitals, Addid Ababa, Ethiopia. Ethiopian Journal of Reproductive Health, Vol. 2 (1).

[31] (MOH and MOPHS) Ministry of Health Services and Ministry of Public Health and Sanitation (2012): National Guidelines for Prevention and Management of Cervical, Breast and Prostate Cancers.

[32] Fisher, A. A., Laing, J. E. and Strocker, J. E. (1998). Handbook for Family Planning, Operation Research Design in Sampling. Population Council, 40-45.

[33] Mupepi, Sylvia C.; Sampselle, Carolyn M.; Johnson, Timothy R. B. (2011). "Knowledge, Attitudes, and Demographic Factors Influencing Cervical Cancer Screening Behavior of Zimbabwean Women." Journal of Women's Health, Vol. 20 (6): 943-952.

[34] Pierre Marie Tebeu, Catherine Mc Carey, David Pirek, Michel Boulvain, Anderson Sama Doh, and Patrick Petignat (2011). Awareness of HPV and cervical cancer prevention among Cameroonian healthcare workers. BMC Womens Health. Vol. 11: 45. doi: 10.1186/1472-6874-11-45.

[35] Austin, L. T., F. Ahmad, M.-J. McNally and D. E. Stewart, (2002) Breast and cervical cancer screening in hispanic women: A literature review using the health belief model. Women's health issues: official publication of the Jacobs Institute of Women's Health, Vol. 12 (3): 122. 